\title{
Lyme Borreliosis - a Multisystem Disease
}

\author{
Angela Revelas ${ }^{1}$, Emmanuel Liannos ${ }^{2}$, Dimitrios Arvanitakis ${ }^{3}$ \\ ${ }^{1}$ Pathological Department of St.Nicolaos-Crete, Greece \\ ${ }^{2}$ Urological Department of St.Nicolaos-Crete, Greece \\ ${ }^{3}$ Neurosurgery Clinic of Venizeleio General Hospital-Crete, Greece
}

Corresponding Author Dr.Angela Revelas

Mobile:

6943503095

E mail:

donnoiko@gmail.com

Key words: Disease ,tick bite, forestry workers, public health, vaccine
Lyme borreliosis, due to the tick-borne spirochete Borrelia burgdorferi sensu lato (Bb.sl), causes significant morbidity throughout the world. Preliminary studies have indicated the presence of the arthropod vector and the pathogen in North Africa. A few clinical cases have been reported. Our objective is to evaluate whether Lyme borreliosis constitutes a threat to public health. To access our objective, we plan to establish a map of the tick distribution, to evaluate the prevalence of Borrelia infection in ticks, to identify Borrelia strains and to determine their genetic diversity, to

\section{INTRODUCTION}

Lyme borreliosis is a multisystem disease involving the skin, nervous system, joints and heart [1]. The causative agent Borrelia burgdorferi is transmitted by tick bite [2]. The prevalence of specific antibodies of blood donors living in endemic areas as described in south Lower Saxony (Germany) has been 7\% and of forestry workers $21 \%$, indicating that this is the most prevalent arthropodborne infection in this geographic region $[3,4]$. One third of flagged Ixodes ricinus contain B.burgdorferi as demonstrated by polymerase chain reaction (PCR) [5]. Therefore reinfection should not be a rare event. Forestry workers with a high risk of repeated tick bites often have a high prevalence and high titers of specific antibodies but only sporadic corresponding clinical symptoms of Lyme borreliosis [6-8]. Thus it has been concluded that a natural protection existed in most cases after an infection with the spirochete.

\section{HISTORY}

In 1980, Steere et al., began to test antibiotic regimens in adult patients identify reservoirs used to maintain Bb.sl, and to evaluate the incidence of the disease in the human population. The knowledge of the natural enzootic cycle involving arthropods and wild vertebrates in the maintenance of Bb.sl should permit to develop prevention strategies to counter any public health threat.

with Lyme disease [9]. In the same year, New York State Health Dept. epidemiologist Jorge Benach provided Willy Burgdorfer, a researcher at the Rocky Mountain Biological Laboratory, with collections of $I$. dammini from Shelter Island, NY, a known Lyme-endemic area as part of an ongoing investigation of Rocky Mountain spotted fever. In examining the ticks for rickettsiae, Burgdorfer noticed "poorly stained, rather long, irregularly coiled spirochetes'. Further examination revealed spirochetes in $60 \%$ of the ticks. Burgdorfer subsequently confirmed his discovery by isolating from patients with Lyme disease spirochetes identical to those found in ticks [10]. In June 1982, he published his findings in Science, and the spirochete was named Borrelia burgdorferi in his honor [2].

\section{PATHOPHYSIOLOGY}

Borrelia burgdorferi can spread throughout the body during the course of the disease, and has been found in the skin, heart, joint, peripheral nervous system and central nervous system. Many of the signs and symptoms of Lyme disease are a 
consequence of the immune response to the spirochete in those tissues [11]. B.burgdorferi is injected into the skin by the bite of an infected Ixodes tick. Tick saliva, which accompanies the spirochete into the skin during the feeding process, contains substances that disrupt the immune response at the site of the bite [12]. This provides a protective environment where the spirochete can establish infection. The spirochetes multiply and migrate outward within the dermis. Days to weeks following the tick bite, the spirochetes spread via the blood stream to joints, heart, nervous system, and distant skin sites, where their presence gives rise to the variety of symptoms of disseminated disease. The spread of B.burgdorferi is aided by the attachment of the host protease plasmin to the surface of the spirochete. If untreated, the bacteria may persist in the body for months or even years, despite the production of B.burgdorferi antibodies by the immune system. In the brain, B.burgdorferi may induce astrocytes to undergo astrogliosis, which may contribute to neurodysfunction [13]. The spirochetes may also induce host cells to secrete products toxic to nerve cells, including quinolinic acid and the cytokines IL-6 and TNF-alpha, which can produce fatigue and malaise $[14,15]$.

In Lyme encephalopathy, diffuse white matter pathology can disrupt grey matter connections, and could account for deficits in attention, memory, visuospatial ability, complex cognition, and emotional status. White matter disease may have a greater potential for recovery than gray matter disease, perhaps because neuronal loss is less common.

\section{EPIDEMIOLOGY}

In northern Africa, B.burgdorferi sensu lato has been identified in Morocco,Algeria, Egypt and Tunisia [16-18]. Lyme disease in Sub-Saharan Africa is presently unknown, but evidence indicates it may occur in humans in this region. The abundance of hosts and tick vectors would favor the establishment of Lyme infection in Africa. In East Africa, two cases of Lyme disease have been reported in Kenya [19].

\section{SIGNS AND SYMPTOMS}

Lyme disease can affect multiple body systems and produce a range of symptoms. Not all patients with Lyme disease will have all symptoms, and many of the symptoms are not specific to Lyme disease, but can occur with other diseases as well. The incubation period from infection to the onset of symptoms is usually one to two weeks, but can be much shorter, or much longer. Symptoms most often occur from May through September, because the nymphal stage of the tick is responsible for most cases. Asymptomatic infection may be much more common among those infected in Europe.

\section{DIAGNOSIS}

Patients with $B$. burgdorferi sensu lato infection may experience one or more clinical syndromes of early or late LB. Usually, early infection consists of localized erythema migrans (EM), which may be followed within days or weeks by clinical evidence of disseminated infection that may affect the skin, nervous system, heart, or joints and subsequently, within months, by late infection [20-23]. EM is the characteristic sign of early infection with $B$. burgdorferi sensu lato and the clinical hallmark of LB. In recent series it is recognized in at least $80 \%$ of patients with objective clinical evidence of $B$. burgdorferi sensu lato infection who meet the CDC surveillance definition of LB [24]. The rash begins at the site of the tick bite as a red macule or papule, rapidly enlarges, and sometimes develops central clearing. The clinical diagnosis of early LB with EM relies on recognition of the characteristic appearance of a skin lesion of at least $5 \mathrm{~cm}$ in diameter. Hematogenous dissemination of $B$. burgdorferi sensu lato to the nervous system, joints, heart, or other skin areas, and occasionally to other organs, may give rise to a wide spectrum of clinical manifestations of what is called early LB. Usually, patients with objective evidence of dissemination experience one or more of the following syndromes: multiple EM lesions, atrioventricular conduction defects, myopericarditis, arthritis, facial palsy, meningitis, and meningoradiculoneuritis (Bannwarth's syndrome) [25-27].

\section{Labotary Diagnosis}

A variety of laboratory techniques have been developed for direct detection of B. burgdorferi sensu lato. These assays provide evidence for the presence of intact spirochetes or spirochete components such as DNA or protein in tick vectors, reservoir hosts, or patients.

Four different approaches have been used in the clinical laboratory: microscope-based assays, detection of $B$. burgdorferi-specific proteins or nucleic acids, and culture. Of these, culture of $B$. 
burgdorferi sensu lato undoubtedly offers the best confirmation of active infection and has been increasingly used as a diagnostic modality by many researchers on both sides of the Atlantic. The availability of cultured organisms has also allowed investigation of the structural, molecular, antigenic, and pathogenetic properties of the different B. burgdorferi sensu lato species.

Direct microscopic detection of B. burgdorferi sensu lato has limited clinical utility in laboratory confirmation of LB due to the sparseness of organisms in clinical samples [2833] Antigen detection assays (aside from PCR) also suffer from the same limitations as microscopic detection. Although antigen capture tests have been used to detect $B$. burgdorferi sensu lato antigens in CSF of patients with neuroborreliosis [34,35] and in urine samples from patients with suspected LB [36], their reliability is poor or at best questionable [37].

\section{Prevention of tick bites}

The ticks that can transmit Lyme disease are found in wooded areas, high grasses, marshes, gardens, and beach areas. In endemic residential areas, clearing brush and trees, removing leaf litter and woodpiles, and keeping grass mowed may reduce tick exposure by removing habitats suitable for ticks and their reservoir hosts [38]. Area application of pesticides to residential properties is effective for suppressing vector ticks but may be harmful to other wildlife and people [39]. Exclusion of deer from residential yards by fencing and maintaining tick-free pets also may reduce tick exposure [40]. Heavily infested tick habitats, such as wooded areas, should be avoided if possible. If not possible, then use of wide trails, not straying off the trail, and not sitting on the ground may decrease exposure. Careful attention also should be given to clothing worn in these areas. Clothing should be light-colored to make tick identification easier. Long sleeves and long pants that are tight at the wrists, ankles, and waist, and long pants tucked into light colored socks are preferable. A hat should be worn in densely wooded areas. Persons should be taught to inspect themselves and their children's bodies and clothing daily after possible tick exposure. Special attention should be given to the exposed hairy regions of the body where ticks often attach, including the heads and necks of children.

Because animal studies indicate that transmission of $B$. burgdorferi from infected ticks usually requires a prolonged duration of attachment ( $\geq 48$ hours), ticks should be removed promptly [41,42]. The body of the tick should not be squeezed during removal. It should be grasped with a fine tweezers as close to the skin as possible and removed by gently pulling the tick straight out without twisting motions. If fingers are used to remove ticks, they should be protected with gloves or facial tissue and washed after removal of the tick. Analysis of ticks to determine if they are infected is not indicated because the predictive values of such tests in relation to the development of human disease are unknown.

\section{NEUROLOGIC MANIFESTATIONS}

Myositis is rare and may occur in the early or late stage. It is diagnosed by muscle enzymes, electromyography or biopsy. Myositis is associated with pains and paresis in proximal muscles in particular. Neuritis cranialis is an acute manifestation and may affect all the cranial nerves including the $\mathrm{N}$. olfactorius. Unilateral or bilateral paresis may occur and recur. Mono-or polyradiculitis and meningopolyradiculitis are the neurologic symptoms of borreliosis that occur most frequently, corresponding to the descriptions by Garin, Bujadoux and Bannwarth $[3,4]$. Radiculitis is connected to an elevated protein concentration, and meningitis is accompanied by lymphocystic pleocytosis.

\section{PROGNOSIS}

For early cases, prompt treatment is usually curative. However, the severity and treatment of Lyme disease may be complicated due to late diagnosis, failure of antibiotic treatment, and simultaneous infection with other tick-borne diseases, including ehrlichiosis, babesiosis, and immune suppression in the patient. A metaanalysis published in 2005 found some patients with Lyme disease have fatigue, joint or muscle pain, and neurocognitive symptoms persisting for years, despite antibiotic treatment [7]. Patients with late stage Lyme disease have been shown to experience a level of physical disability equivalent to that seen in congestive heart failure.

\section{Lyme Disease Vaccine}

Animal studies have demonstrated that purified recombinant proteins, particularly of certain outer surface proteins (Osp) of B burgdorferi, 
such as Osp A, B, and C, induce antibody responses that are highly protective [43]. The most extensively studied [44] of the single Osp vaccines and the one currently licensed contains recombinant OspA (rOspA), which is highly protective in the mouse model when the challenge strain is homologous or closely related to the isolate from which the OspA was derived $[45,46]$. However, when the challenge strain is different from the isolate from which the OspA was derived, protection to challenge is minimal or nonexistent.

Route of administration, immunization schedule, and dose:

Three doses of $0.5 \mathrm{~mL}(30 \mu \mathrm{g})$ of rOspA vaccine administered by intramuscular injection are required for optimal protection; the second dose is given 1 month later, and a third dose is given 12 months after the first dose. Dosages should be timed so that the second and the third doses are given several weeks before the start of the Lyme disease transmission season, which usually begins in April.

Preliminary data suggest that other immunization schedules (e.g., 0, 1, 6 months) are safe and induce antibody responses similar to the $0,1,12$ month schedule [46]. However, at this time, only the $0,1,12$ month schedule is approved by the US Food and Drug Administration.

\section{CONCLUSION}

Borrelia is transmitted to humans by the bite of infected ticks belonging to a few species of the genus Ixodes. Delayed or inadequate treatment can lead to the more serious symptoms, which can be disabling and difficult to treat. At present the major problem in the early diagnosis of borreliosis is the high percentage of seronegativity of $20-50 \%$ depending on the duration of the erythema migrans. Another problem is the persistence of elevated IgM antibodies after therapy. Misinterpretations of serology contribute to the over diagnosis and over treatment of chronic Lyme disease and irrational Lyme anxiety. The diagnosis of Lyme borreliosis should primarily be based on clinical and epidemiologic evidence.

\section{REFERENCES}

1. Steere AC. Medical progress-Lyme disease. N.Engl. J.Med. 1989; 321: 586-596.
2. Burgdorfer W, Barbour AG, Hayes SF, Benach JL, Grunwaldt E, Davis JP. Lyme disease-a tickborne spirochetosis? Science. 1982 Jun 18;216(4552):1317-9.

3. Eiffert H, Ohlenbusch A, Fehling W, Lotter H, Thommssen R. Nucleotide sequence of the ospABoperon of a Borrelia burgdorferi strain expressing OspA but not OspS. Infect Immun. 1992; 60 :1864-1868.

4. Christen HJ, Hanefeld F, Eiffert H, Thomssen R. Epidemiology and clinical manifestations of Lyme borreliosis in childhood. A prospective multicentre study with special regard to neuroborreliosis. Acta Paediatr. Scand.1993; Suppl. 386: 1-75.

5. Eiffert H, Ohlenbusch A, Christen HJ, Thomssen R, Spielman A, Matuschka FR. Nondifferentiation between Lyme disease spirochetes from vector ticks and human cerebrospinal fluid. $J$ Infect Dis. 1995;171(2):476-9.

6. Hassler D, Maiwald M. Re-Infektionen mit Borrelia burgdorferi bei einem immunkompetensen Patient. Dutche Med. Wochenschr. 1994 ; 119 :338-342

7. Fahrer H, Sauvain MJ, vd Linden S, Zhioua E, Gern L, Aeschlimann A. [Prevalence of Lyme borreliosis in a Swiss population at risk]. Schweiz Med Wochenschr. 1988 16;118(2):65-9.

8. Münchhoff P, Wilske B, Preac-Mursic V, Schierz G. Antibodies against Borrelia burgdorferi in Bavarian forest workers. Zentralbl. Bakteriol Mikrobiol Hyp.A.1987; 263:412-419.

9. Steere AC, Hutchinson GJ, Rahn DW, Sigal LH, Craft JE, DeSanna ET. Treatment of the early manifestations of Lyme disease. Ann Intern.Med 1983 ;99(1): 22-26.

10. Burgdorfer W. Discovery of the Lyme disease spirochete and its relation to tick vectors. Yale $J$ Biol Med. 1984;57(4):515-20.

11. Auwaerter PG, Aucott J, Dumler JS. Lyme borreliosis (Lyme disease) molecular and cellular pathobiology and prospects for prevention, diagnosis and treatment. Expert Rev Mol. Med. 2004; 6(2): 1-22.

12. Fikrig E, Narasimhan S. Borrelia burgdorferitraveling incognito? Microbes. Infect. 2006; 8(5) : 1390-9.

13. Ramesh G, Alvarez AL, Roberts ED, Dennis VA, Lasater BL, Alvarez X. Pathogenesis of Lyme neuroborreliosis. Borrelia burgdorferi lipoproteins induce both proliferation and apoptosis in rhesus monkey astrocytes. Eur. J. Immunol. 2003; 33(9) : 2539-50. 
14. Halperin JJ, Heyes MP. Neuroactive kynurenines in Lyme borreliosis. Neurology 1992; 42(1) : 43-50.

15. Papanicolaou DA, Wilder RL, Manolagas SC, Chrousos GP. The pathophysiologic roles of interleukin-6 in human disease. Ann. Intern. Med $1998 ; 128(2)$ : 127-137.

16. Bovattour A, Ghorbel A, Chabchoub A, Postic D. Lyme borrerliosis in North Africa. Arch Inst Pasteur Tunis. 2004;81(1-4):13-20.

17. Dsouli N, Younsi-Kabachii H, Postic D, Nouiras S, Gerne L. Reservoir role of lizard Psammodromus algirus in transmission cycle of Borrelia burgdorferi sensu lato in Tunisia. J.Med.Entomol. 2006; 43(4) : 737-742.

18. Helmy N. Seasonal abundance of Ornithodoros, savignyi and prevalence of infection with Borrelia spirochetes in Egypt. J Egypt Soc Parasitol. 2000;30(2):607-19.

19. Javi JO, Gathua SN. Lyme disease report of two cases. East Afr Med J. 2005;82(5):267-9.

20. Nadelman RB, Wormser GP. Lyme borreliosis. Lancet $1998 ; 352: 557-565$.

21. Stanek G, Strle F. Lyme borreliosis. Lancet 2003; 362:1639-1647.

22. Steere AC. Lyme disease. N. Engl. J. Med. 2001; 345:115-125.

23. Steere, AC, Batsford WP, Weinberg M, Alexander $\mathrm{J}$, Berger $\mathrm{HJ}$, Wolfson $\mathrm{S}$ et al. Lyme carditis: cardiac abnormalities of Lyme disease. Ann. Intern. Med. 1980; 93:8-16.

24. Steere AC, Sikand VK, Meurice F, Parenti DL, Fikrig E, Schoen RT, et al. Vaccination against Lyme disease with recombinant Borrelia burgdorferi outer-surface lipoprotein A with adjuvant. N. Engl. J. Med. 1998; 339:209-215.

25. Oschmann P, Dorndorf W, Hornig C, Schäfer C, Wellensiek HJ, Pflughaupt KW. Stages and syndromes of neuroborreliosis. J. Neurol. 1998; 245:262-272.

26. Strle F, Nelson JA, Ruzic-Sabljic E, Cimperman J, Maraspin V, Lotric-Furlan S, et al. European Lyme borreliosis: 231 culture-confirmed cases involving patients with erythema migrans. Clin. Infect. Dis. 1996; 23:61-65.

27. van der Linde MR. Lyme carditis: clinical characteristics of 105 cases. Scand. J. Infect. Dis. 1991; Suppl. 77:81-84.

28. Berger BW, Clemmensen OJ, Ackerman AB. Lyme disease is a spirochetosis. A review of the disease and evidence for its cause. Am. J. Dermatopathol. 1983; 5:111-124.
29. Bergler-Klein J, Sochor H, Stanek G, Globits S, Ullrich R, Glogar D. Indium 111-monoclonal antimyosin antibody and magnetic resonance imaging in the diagnosis of acute Lyme myopericarditis. Arch. Intern. Med. 1993; 153:2696-2700

30. de Koning J, Bosma RB, Hoogkamp-Korstanje JA. Demonstration of spirochaetes in patients with Lyme disease with a modified silver stain. $J$. Med. Microbiol. 1987; 23:261-267.

31. de Koning J, Duray PH. Histopathology of human Lyme borreliosis 1993, p. 70-92. In K. Weber and W. Burgdorfer (ed.), Aspects of Lyme borreliosis. Springer-Verlag, Berlin, Germany.

32. de Koning J, Hoogkamp-Korstanje JA, van der Linde MR, Crijns HJ. Demonstration of spirochetes in cardiac biopsies of patients with Lyme disease. J. Infect. Dis. 1989; 160:150-153.

33. Hoffmann JC, Stichtenoth DO, Zeidler $H$, Follmann M, Brandis A, Stanek G, et al. Lyme disease in a 74-year-old forest owner with symptoms of dermatomyositis. Arthritis Rheum. 1995; 38:1157-1160

34. Courtney JW, Massung RF. Multiplex Taqman PCR assay for rapid detection of Anaplasma phagocytophila and Borrelia burgdorferi. Ann. N. Y. Acad. Sci. 2003; 990:369-370.

35. Coyle PK, Deng Z, Schutzer SE, Belman AL, Benach J, Krupp LB, et al. Detection of Borrelia burgdorferi antigens in cerebrospinal fluid. Neurology 43:1093-1098.

36. Dorward DW, Schwan TG, Garon CF. Immune capture and detection of Borrelia burgdorferi antigens in urine, blood, or tissues from infected ticks, mice, dogs, and humans. J Clin Microbiol. 1991;29(6):1162-1170.

37. Klempner MS, Schmid CH, Hu L, Steere AC, Johnson G, McCloud B, et al. Intralaboratory reliability of serologic and urine testing for Lyme disease. Am. J. Med. 2001; 110:217-219.

38. Schulze TL, Jordan RA, Hung RW. Suppression of subadult Ixodes scapularis (Acari: Ixodidae) following removal of leaf litter. J Med Entomol. 1995; 32:730-733.

39. Curran KL, Fish D, Piesman J. Reduction of nymphal Ixodes dammini (Acari: Ixodidae) in a residential suburban landscape by area application of insecticides. J Med Entomol. 1993; 30:107113.

40. Hayes EB, Maupin GO, Mount GA, Piesman J. Assessing the effectiveness of local Lyme disease control. J Public Health Manage Pract. 1999; 5:86-94. 
41. Piesman J. Dynamics of Borrelia burgdorferi transmission by nymphal Ixodes dammini ticks. $J$ Infect Dis. 1993; 167:1082-1085.

42. Piesman J, Mather TN, Sinsky RJ, Spielman A. Duration of tick attachment and Borrelia burgdorferi transmission. J Clin Microbiol. 1987; 25:557-558.

43. Wormser GP. Lyme disease vaccine. Infection. 1996; 24:203-207.

44. Fikrig E, Barthold SW, Kantor FS, Flavell RA. Protection of mice against the Lyme disease agent by immunizing with recombinant Osp A. Science. 1990; 250:553-556.

45. Schaible UE, Kramer MD, Eichmann K, Modolell M, Museteanu C, Simon MM. Monoclonal antibodies specific for the outer surface protein A (OspA) of Borrelia burgdorferi prevent Lyme borreliosis in severe combined immunodeficiency (SCID) mice. Proc Natl Acad Sci U S A. 1990; 87:3768-3772.

46. Van Hoecke C, Lebacq E, Beran J, Perenti D. Alternative vaccination schedules $(0,1,6$ months versus $0,1,12$ months) for a recombinant OspA Lyme disease vaccine. Clin Infect Dis. 1999; 28:1260-1264.

Peer Reviewer: Mohamad Emara ;Lecturer of Tropical Medicine, Zagazig University, Egypt. Editor :Tarik Zaher; Assistant Professor of Tropical Medicine, Zagazig University, Egypt 